\title{
OPTIMIERUNG ALLER MAßNAHMEN ZUR DECKUNG DES KLASSENRAUMBEDARFS AN HAMBURGER SCHULEN
}

\author{
S. Fleischmann, CAP debis GEI GmbH, Hamburg
}

In den letzten Jahren sind in der Stadt Hamburg aufgrund rückläufiger Schülerzahlen Kapazitäten an Klassenräumen abgebaut worden. Mittlerweile haben sich die Voraussetzungen geändert. Die Behörde für Schule, Jugend und Berufsbildung (BSJB) geht davon aus, daß bis zum Jahre 2005 mit einem Zuwachs in Höhe von ca. 15 Prozent zu rechnen ist. Erschwert wird das Problem dadurch, daß der Schülerzuwachs nicht homogen auf alle Stadtteile, Schulformen und Klassenstufen verteilt ist. Es ist damit zu rechnen, daß an bestimmten Schulen bis zu 30 Prozent mehr Schüler angemeldet werden.

Deckung des zusätzlichen Klassenraumbedarfs durch Neubau ist kostspielig und nicht immer möglich. Es gilt daher, die vorhandenen Kapazitäten optimal zu nutzen und Erweiterungsmaßnahmen möglichst zu vermeiden. Dabei ist eine für alle Schulen "akzeptable" Lösung zu finden.

Zur Deckung des Raumbedarfs sind folgende Maßnahmen möglich:

- Vorübergehende Bereitstellung von Räumen in Überschuß-Schulen für MangelSchulen (Raumverfügungen)

- Reaktivierung stillgelegter, artfremd genutzter oder vermieteter Räume

- Neubau von Pavillons oder festen Gebäuden

Für diese Maßnahmen gibt es eine ganze Reihe von einschränkenden Bedingungen. Die wichtigsten davon sind:

- Raumverfügungen sind nur zwischen bestimmten Schulen zulässig. Neben geographischer Nachbarschaft sind dabei weitere Bedingungen, die schwer in Regeln zu fassen sind, von Bedeutung.

- Im Falle einer Raumverfügung ist der Mangel-Schule eine Mindestanzahl von Klassenräumen zu überlassen.

- Um eine zu starke Zersplitterung zu verhindern, ist eine maximale Anzahl von Standorten für die einzelnen Schulen nicht zu überschreiten.

Aufgrund der Vielzahl an Handlungsalternativen und der komplexen Randbedingungen ist manuell eine optimale Lösung kaum zu finden. Die mathematische Formulierung aller Anforderungen führt aber auf ein gemischt ganzzahliges Optimierungsproblem, das für die automatisierte Lösung durch einen Rechner gut geeignet ist.

Dazu wird die Standard-Optimierungs-Software SCICONIC eingesetzt. Einerseits erleichtert SCICONIC dem OR-Fachmann die Eingabe des mathematischen Modells während der Entwicklungsphase, andererseits läßt sich SCICONIC mit seinem SQL-Interface gut in ein dialogorientiertes, anwenderfreundliches Software-System einbinden. Dies ist von besonderer Bedeutung, da die Optimierung der durchzuführenden Maßnahmen vor jedem Schuljahr durch Planer der BSJB erfolgt, die weder DV- noch OR-Erfahrung besitzen. 